\title{
A-Priori Validation of Scalar Dissipation Rate Models for Turbulent Non-Premixed Flames
}

\author{
M. P. Sitte ${ }^{1}$ (D) $\cdot$ C. Turquand d'Auzay ${ }^{2} \cdot$ A. Giusti $^{3}$ (D) $\cdot$ E. Mastorakos ${ }^{1}$ (D) $\cdot$ \\ N. Chakraborty ${ }^{2}$ (D)
}

Received: 15 June 2020 / Accepted: 9 September 2020 / Published online: 14 October 2020

(c) The Author(s) 2020

\begin{abstract}
The modelling of scalar dissipation rate in conditional methods for large-eddy simulations is investigated based on a priori direct numerical simulation analysis using a dataset representing an igniting non-premixed planar jet flame. The main objective is to provide a comprehensive assessment of models typically used for large-eddy simulations of nonpremixed turbulent flames with the Conditional Moment Closure combustion model. The linear relaxation model gives a good estimate of the Favre-filtered scalar dissipation rate throughout the ignition with a value of the related constant close to the one deduced from theoretical arguments. Such value of the constant is one order of magnitude higher than typical values used in Reynolds-averaged approaches. The amplitude mapping closure model provides a satisfactory estimate of the conditionally filtered scalar dissipation rate even in flows characterised by shear driven turbulence and strong density variation.
\end{abstract}

Keywords Scalar dissipation rate $\cdot$ Large-eddy simulation $\cdot$ Conditional moment closure Non-premixed flames

\section{Introduction}

Any combustion process with imperfectly mixed reactants requires mixing at the molecular level of fuel and oxidiser, as well as energy transport from the reacting region to the unburnt reactants, for the flame to develop. In modelling of turbulent non-premixed flames, mixing at the molecular level (also called micro-mixing) is generally related to the energy cascade of a conserved scalar, the mixture fraction $\xi$, and typically enters into models through the terms representing the dissipation of scalar fluctuations at unresolved scales.

\section{A. Giusti}

a.giusti@imperial.ac.uk

1 Hopkinson Laboratory, Department of Engineering, University of Cambridge, Cambridge CB2 1PZ, UK

2 School of Engineering, Newcastle University, Newcastle-Upon-Tyne NE1 7RU, UK

3 Department of Mechanical Engineering, Imperial College London, South Kensington Campus, London SW7 1AL, UK 
The scalar dissipation rate (SDR), defined as $N_{\xi}=D \nabla \xi \cdot \nabla \xi$, represents the local rate of mixing (diffusion) at molecular level (Bilger 2004) and therefore it is a key element for the modelling of turbulence effects on reaction rates. In presumed PDF models, the SDR controls the rate at which the variance of the resolved conserved scalar decays in time; the SDR also affects directly the local flame structure in advanced turbulent combustion models such as the conditional moment closure (CMC) (Klimenko and Bilger 1999) as well as flamelet-based approaches (Peters 1984). In models based on transported PDF (Pope 1985) as well as in the Multiple Mapping Conditioning (MMC) (Klimenko and Pope 2003), the micro-mixing effects are included through mixing models (Ren and Pope 2004; Varna et al. 2017) with physical meaning equivalent to the SDR. Therefore, the modelling of SDR can also be relevant to such modelling approaches.

The focus of this study is on the modelling of SDR in large-eddy simulations (LES) and the CMC model for turbulent non-premixed flames. In this context, the interest is on the modelling on the Favre-filtered SDR, $\widetilde{N}_{\xi}$, and the conditionally filtered SDR, indicated in the following as $\left\langle\widetilde{N_{\xi} \mid \eta}\right\rangle$, where $\eta$ is the sample space variable of the mixture fraction. The singly-conditioned LES-CMC approach has been used so far for a large variety of nonpremixed flame configurations showing successful prediction of challenging flame phenomena characterised by strong turbulence chemistry interactions (low Da number) such as local extinctions (Zhang et al. 2015) and flame blow-out (Zhang and Mastorakos 2016). However, such work has also shown a strong dependence of the results on the scalar dissipation rate modelling (Garmory and Mastorakos 2011), which affects the local fluctuations of the flame structure and flame transients in general. LES-CMC has also been shown to be able to predict the ignition transient of turbulent jet flames (Zhang et al. 2019). In this case, the small-scale strain rate that affects the SDR is important for the prediction of the kernel ignition probability (Richardson and Mastorakos 2007). Furthermore, although kernel growth is mainly driven by chemistry and transport in physical space terms, SDR modelling becomes important for the prediction of the subsequent edge flame propagation and flame stabilisation (Hesse et al. 2009; Turquand d'Auzay et al. 2019a). Micro-mixing affects the spreading of the flame across mixture fraction iso-surfaces and is also important for the lift-off height of the stabilised flame (Navarro-Martinez and Kronenburg 2011; Zhang et al. 2019). An accurate and reliable model of both the resolved and the conditional SDR is therefore crucial for improving the predictive capabilities of LES-CMC and also for capturing ignition and finite-rate effects reliably in turbulent combustion. It should be noted that the availability of reliable models for SDR will be of great benefit also to all the other approaches that require modelling of micro-mixing through the SDR, e.g. all the approaches that solve for the sub-grid scale mixture fraction variance.

Modelling of the filtered SDR includes contributions from both the resolved and subgrid scales, $\widetilde{N}_{\xi}=\widetilde{N}_{\xi, \text {,es }}+\widetilde{N}_{\xi, \text { sgs }}$. The resolved part of the SDR is typically modelled as $\widetilde{N}_{\xi \text {,res }}=\tilde{D} \nabla \tilde{\xi} \cdot \nabla \tilde{\xi}$. For the sub-grid part, $\widetilde{N}_{\xi, \text { sgs }}=D_{\text {sgs }} \nabla \tilde{\xi} \cdot \nabla \tilde{\xi}$ has been proposed, based on spectral arguments or equilibrium assumption (Girimaji and Zhou 1996; Pierce and Moin 1998). Yet, this model is not consistent with the transport equation for $\xi^{\prime \prime 2}$, since it cancels with the production term (Jiménez et al. 2001). Alternatively, the assumption of linear relaxation in a characteristic eddy turnover time has been proposed (Jiménez et al. 2001; Branley and Jones 2001), where the sub-grid SDR is proportional to the sub-grid scale variance of the conserved scalar divided by the turbulent time scale, $\widetilde{N}_{\xi \text {,sgs }} \sim \widetilde{\xi}^{\prime \prime 2} / \tau_{T}$, typically expressed as $\widetilde{N}_{\xi \text {,sgs }}=(1 / 2) C_{N} v_{\text {sgs }} \widetilde{\xi}^{\prime \prime 2} / \Delta^{2}$ or equivalent forms $\left(v_{\mathrm{sgs}}\right.$ is the sub-grid kinematic viscosity and $\Delta$ is the LES filter size). The linear relaxation model has been used in a large variety of approaches, ranging from flamelet to conditional methods. Despite its importance, the linear relaxation model has never been extensively validated at relevant 
flame conditions and several different values have been proposed so far for the constant of proportionality $C_{N}$. Such values are mainly based on ad-hoc choices rather than being the results of validation against measurements or direct numerical simulations (DNS). Although a number of papers focused on the modelling of SDR, most of these concentrated on either SDR modelling for reactive scalars in premixed flames or use model constants typically used in the RANS context. Previous work on the modelling of the SDR in the LES context usually focuses on dynamic procedures (e.g. Kaul et al. (2009), Kaul and Raman (2011), Kaul et al. (2013)). Nevertheless, the SDR models used in combination with CMC or flamelets often use constant model parameters with no dynamic evaluation. In a few papers (Jiménez et al. 2001; Kaul et al. 2009) the information of the modelling constant, can be extracted, but these studies have been conducted for non-reacting flows. Therefore, this work attempts to provide a reliable value of modelling constant $C_{N}$ in a flow configuration that represents an igniting jet (transient flame) which is characterised by both evolving mixture fraction and density gradients.

The lack of validation in reacting conditions is even more prominent when models for the conditionally filtered SDR are considered. The conditionally filtered SDR represents the rate of mixing from the resolved scales down to molecular level in the conditional space. Validation requires the availability of data for mixture fraction gradients conditioned on $\eta$. Several models have been been proposed so far to model the conditional SDR, including the amplitude mapping closure (AMC) (O'Brien and Jiang 1991) and models derived from the PDF transport equations (Girimaji 1992; Kronenburg et al. 2000; Devaud et al. 2004). These models were originally developed for RANS applications whereas, in LESCMC, much simpler approaches have been pursued (Navarro-Martinez et al. 2005; Triantafyllidis and Mastorakos 2010), based on the assumption that conditional moments change slowly in space and therefore using conditional volume averaging. These models have not been validated yet against DNS data and the present paper focuses on different implementations of the models that are in practical use.

In this work, the modelling of SDR in LES context is investigated by means of an a priori analysis based on a three-dimensional DNS dataset of an igniting planar jet flame. The focus is on the modelling of both the Favre-filtered SDR and the conditionally filtered SDR. The objectives are: (i) to assess the modelling of Favre-filtered SDR based on linear relaxation and determine the optimal value of the constant $C_{N}$; (ii) to evaluate different formulations for the conditionally-filtered SDR.

\section{Methods}

\subsection{DNS Dataset}

A three-dimensional DNS dataset of a planar turbulent igniting methane-air jet has been considered. The case is taken from a larger database (Turquand d'Auzay and Chakraborty 2020), which has been used to analyse the effects of the location of the ignitor in terms of the mean mixture composition, flammability factor and mixture fraction gradient on localised forced ignition of a planar turbulent jet.

A two-step chemical mechanism was used for its low computational cost and the ability to accurately estimate the laminar burning velocity across the flammability range when preexponential adjustment is applied for rich mixtures (Bibrzycki and Poinsot 2010; Turquand d'Auzay et al. 2019a). The use of simple chemistry is justifiable by previous findings and 
the scope of the present study: the validity of single-step chemistry compared to detailed chemistry in terms of strain rate and curvature dependence of displacement speed in edge flames, vitally important for the flame propagation in this configuration, has been shown in previous DNS studies (Chakraborty and Mastorakos 2006; Hesse et al. 2009). The present mechanism further includes the $\mathrm{CO}+0.5 \mathrm{O}_{2} \longleftrightarrow \mathrm{CO}_{2}$ reaction, which allows for better prediction of the flow expansion around the flame (Peters and Williams 1987). The behaviour of the reduced two-step mechanism in comparison to detailed chemical mechanisms (e.g. GRI-3.0) has been presented elsewhere (Turquand d'Auzay et al. 2019a) and thus is not repeated here for the sake of brevity. The current investigation focuses on the modelling of SDR of mixture fraction, which is primarily driven by micro-mixing statistics and not by the chemistry and thus the choice of chemical mechanism is unlikely to affect the SDR statistics presented here.

The simulations have been carried out using the well-known DNS code SENGA+ (Jenkins and Cant 1999). The spatial discretisation and time advancement have been carried out using high order finite-difference (10th order for the internal grid points and gradually reducing to 2 nd order at the non-periodic boundaries) and 3rd order explicit RungeKutta schemes. The slot jet width was $h=7.8 \delta_{\text {th }}$ where $\delta_{\text {th }}=\left[T_{\text {ad }}-T_{0}\right] / \max |\nabla \hat{T}|_{L}$ is the thermal flame thickness of the stoichiometric mixture with $T_{\mathrm{ad}}, T_{0}$ and $\hat{T}$ being the adiabatic flame temperature of the stoichiometric mixture, unburned gas temperature and instantaneous dimensional temperature, respectively. The simulation domain was $L_{x} \times L_{y} \times L_{z} \approx 37 h \times 19 h \times 4.3 h$, which is discretised using a uniform Cartesian grid of $1920 \times 990 \times 225$ ensuring at least 7 grid points within $\delta_{\text {th }}$. This grid also ensures that the Kolmogorov length scale $\eta_{K}$ remains about 1.2 times the grid spacing. The boundaries in the lateral directions (i.e. $y$ and $z$ directions) are periodic and the boundaries in the streamwise direction are taken to be partially non-reflecting. $\mathrm{A} \mathrm{CH}_{4}$-air mixture is injected through the slot inlet with a bulk mean velocity of $U_{\mathrm{j}}=23.5 S_{\mathrm{L}}^{0}$, whereas the coflow velocity of pure air is $U_{\mathrm{c}}=0.1 U_{\mathrm{j}}=2.35 S_{\mathrm{L}}^{0}$. This yields a jet Reynolds number of $\operatorname{Re}_{\mathrm{j}}=\rho_{0} U_{\mathrm{j}} h / \mu=650$. The co-flow is assumed to be laminar, whereas the velocity fluctuations at the slot inlet are imposed by scanning a plane through a frozen periodic turbulent channel flow; turbulent velocity fluctuations normalised by the mean flow velocity are taken from fully developed channel flow with a frictional Reynolds number of $\operatorname{Re}_{\tau}=395$.

The mixture injected through the slot is composed of $27.5 \%$ of $\mathrm{CH}_{4}$ and $72.5 \%$ of air by volume, whereas the oxidiser mass fraction in air is $Y_{\mathrm{O} \text {,air }}=0.233$. The values of $\xi$ corresponding to the stoichiometric, lean and rich flammability limits are $\xi_{\mathrm{st}}=0.317, \xi_{1}$ $=0.19$ and $\xi_{\mathrm{r}}=0.49$, respectively. The unburned gas temperature is $T_{0}=415 \mathrm{~K}$, which yields a heat release parameter of $\tau=\left(T_{\mathrm{ad}}-T_{0}\right) / T_{0}=4.5$. Standard values are taken for the Prandtl and Schmidt numbers $(\mathrm{Pr}=\mathrm{Sc}=0.7)$ and the ratio of specific heats $(\gamma=1.4)$. A precursor non-reacting flow DNS has been conducted for about two flow-through times (i.e. $2 t_{\mathrm{j}}=4 L_{x} /\left(U_{\mathrm{j}}+U_{\mathrm{c}}\right)$ ) such that the initial transients disappear and that realistic fluctuations of both the mixture composition and velocity fields are obtained. This field is taken to be the initial condition for the reacting flow simulations.

Only the thermal aspect of forced ignition is modelled here by adding a Gaussian source term, $q^{\prime \prime \prime}=A_{\mathrm{sp}} \exp \left[-r^{2} /\left(2 R_{\mathrm{sp}}^{2}\right)\right]$, with $r$ being the distance from the ignitor centre and $R_{\mathrm{sp}}$ representing the characteristic width of energy deposition, to the energy conservation equation, following several previous analyses (Turquand d'Auzay et al. 2019a; Vázquez Espí and Liñán 2002; Chakraborty et al. 2009; Wandel 2014). The constant $A_{\mathrm{sp}}$ in the expression for $q^{\prime \prime \prime}$ is determined by the total ignition power $\dot{Q}=\int_{V} q^{\prime \prime \prime} \mathrm{d} V=a_{\mathrm{sp}} \rho_{0} C_{p} \tau T_{0}\left(4 \pi \delta_{z}^{3} / 3\right)\left[H(t)-H\left(t-t_{\mathrm{sp}}\right)\right] / t_{\mathrm{sp}} \quad$ (Turquand d'Auzay et al. 2019a; Vázquez Espí and Liñán 2002; Chakraborty et al. 2009; Wandel 2014) where $a_{\mathrm{sp}}$ 
is a parameter determining the total energy deposited by the ignitor, $\delta_{z}$ is the Zel'dovich flame thickness of the undiluted stoichiometric mixture $\left(\delta_{z}=D_{0} / S_{L}\right.$, where $D_{0}$ is the mass diffusivity in the unburned reactants) and $H(t)$ and $H\left(t-t_{\mathrm{sp}}\right)$ are Heaviside functions, which ensure that the ignitor is active until $t_{\mathrm{sp}}$. The energy deposition duration $t_{\mathrm{sp}}$ is expressed as $t_{\mathrm{sp}}=b_{\mathrm{sp}} \delta_{z} / S_{L}^{0}$. For the present analysis, $a_{\mathrm{sp}}=10, b_{\mathrm{sp}}=0.34$ and $R_{\text {sp }}=2.45 \delta_{z}$ are used, and the ignitor centre is placed on the jet axis at $x / h=15$, which corresponds to a Favre-mean mixture fraction of $\tilde{\xi}=0.53=1.68 \xi_{\mathrm{st}}$ at the beginning of the energy deposition period (i.e. $t=0$ ). All the SDR statistics presented in this work correspond to a time long after the spark time (i.e. $t \gg t_{\mathrm{sp}}$ ) when the maximum temperature within the domain settles to the adiabatic flame temperature of the stoichiometric mixture and thus it can be safely assumed that the ignition modelling does not affect the statistics and model performances presented in this paper. In total, the reacting flow simulation has been continued for $t / t_{\mathrm{sp}}=80$.

This configuration is qualitatively similar to an experimental configuration (Ahmed and Mastorakos 2006) (however a planar jet is used instead of a round jet in the experiments), which has been used extensively for several subsequent LES studies (e.g. Zhang et al. (2019) and references therein). This DNS dataset is representative of a laboratoryscale configuration and includes the effects of finite-rate chemistry and mean shear in a turbulent environment in contrast to a vast body of literature based on canonical configurations without any mean velocity and shear (Turquand d'Auzay et al. 2019a; Vázquez Espí and Liñán 2002; Chakraborty et al. 2009; Wandel 2014). In the following, spatial coordinates $\left(x^{+}, y^{+}, z^{+}\right)$are normalised by the jet width $h ; \nabla^{+}=\left(\partial / \partial x^{+}, \partial / \partial y^{+}, \partial / \partial z^{+}\right)$; time $t^{+}=t \times\left(U_{\mathrm{j}} / h\right)$; non-dimensional SDR is computed as $N_{\xi}^{+}=\left(1 /\left[\operatorname{Re}_{j} \mathrm{Sc}\right]\right) \nabla^{+} \xi \cdot \nabla^{+} \xi$.

Three snapshots of the normalised mixture fraction field overlaid with iso-temperature contours are shown in Fig. 1 at times corresponding to $t^{+}=10,20$ and 30. At $t^{+}=10$, the kernel has already been advected downstream by the local flow field while the downstream flame front propagation has allowed the kernel to grow in the streamwise direction. According to the experimental observations of Ahmed and Mastorakos (2006), this corresponds to the downstream and radial propagation phase of the ignited hot gas kernel. By $t^{+}=20$, the flame front spans across the whole jet, while pockets of unburned fuel-rich mixture can be seen near the jet centreline. Noting that the flame height at both $t^{+}=20$ and $t^{+}=30$ is comparable, as well as noting the large upstream propagation of the reactive

Fig. 1 Flame at $t^{+} \approx 10,20$ and 30. Surface plot of mixture fraction $\xi / \xi_{\text {st }}$ and black iso-contours of non-dimensional temperature $T=\left(\hat{T}-T_{0}\right) /\left(T_{a d}-T_{0}\right)=0.5$. The spark location is indicated at $\left(x^{+}, y^{+}\right)=(15,0)$

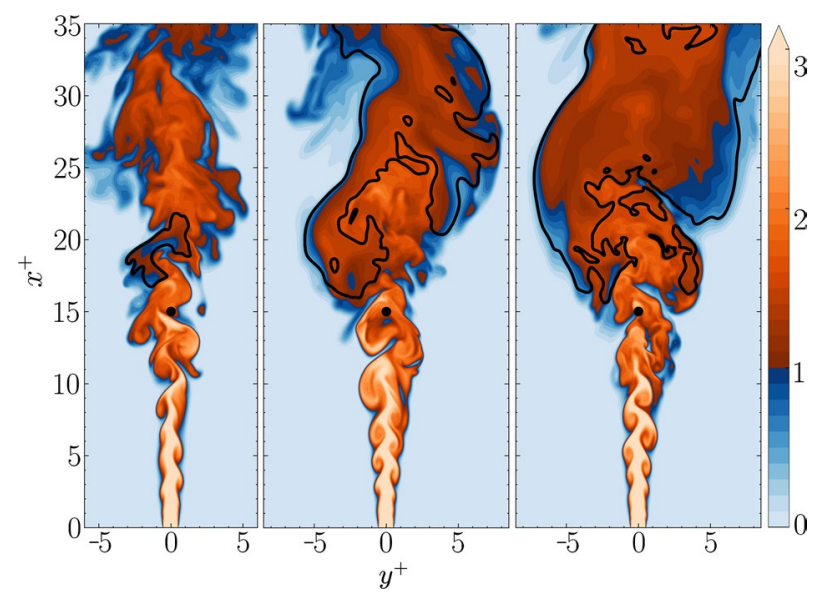


front present in the right shear layer, the onset of flame stabilisation can be observed. Extinction holes can also be observed at late time in the right shear layer.

In the present DNS dataset the jet Reynolds number was kept moderate in the interest of a large computational domain. Nevertheless, the non-isotropic turbulent inflow with the additional presence of wall ejections allows for more realistic flow conditions, than would be possible with the usual practice of superimposing a homogeneous isotropic decaying turbulence onto a parabolic profile or white noise imposed on a laminar flow profile. This was previously discussed in detail by Akselvoll and Moin (1996). The simplification of using periodic span-wise boundary conditions for the sake of computational economy leads to the persistence of Kelvin-Helmholtz instability (Fig. 1). However, the jet appears fully developed by $x^{+} \approx 10$ and cold flow statistics in terms of mean mixture fraction and velocity profiles are in good agreement with experimental data on turbulent jets (Namer and Ötügen 1988) up to the axial length of $x^{+}>30$, which implies that the flame develops and propagates in a region that is representative of realistic turbulent jet flow (Turquand d'Auzay and Chakraborty 2020). While the turbulent Reynolds number that can be achieved using DNS remains modest, previous analyses for premixed turbulent flames suggested that the model parameters for scalar dissipation rate closures attain asymptotic values for relatively small values of $\mathrm{Re}_{\mathrm{t}} \approx 50$ (Chakraborty and Swaminathan 2013).

In terms of generality of the present study, it should further be noted that in turbulent premixed flames, SDR models calibrated for statistically planar flames have been found to remain valid for relatively complex geometrical configurations (Gao and Chakraborty 2016) and that the statistics of mixture fraction gradients in statistically planar turbulent spray flames (Wacks and Chakraborty 2016) are qualitatively similar in planar spray jets (Turquand-d'Auzay et al. 2019b). This inspires confidence that the modelling conclusions drawn in this analysis are unlikely to change in another configuration provided the mean curvature of that configuration remains small.

\subsection{Analysis}

In LES, the sub-grid scale mixing field of the scalar variable, such as the mixture fraction $\xi$, can be described by the filtered probability density function (FDF) (Pope 1991):

$$
p(\eta ; \mathbf{x}, t)=\int_{V} \delta\left(\eta-\xi\left(\mathbf{x}^{\prime}, t\right)\right) G\left(\mathbf{x}-\mathbf{x}^{\prime}\right) \mathrm{d} V^{\prime}
$$

where $G\left(\mathbf{x}-\mathbf{x}^{\prime}\right)$ represents the filter kernel corresponding to a filter of size $\Delta$. The filtered mean of a variable $f$ conditional on $\xi$ is (Bushe and Steiner 1999):

$$
\begin{aligned}
\langle f(\mathbf{x}, t)| \xi(\mathbf{x}, t)= & \eta\rangle= \\
& \frac{\int_{V} f\left(\mathbf{x}^{\prime}, t\right) \delta\left(\eta-\xi\left(\mathbf{x}^{\prime}, t\right)\right) G\left(\mathbf{x}-\mathbf{x}^{\prime}\right) \mathrm{d} V^{\prime}}{p(\eta ; \mathbf{x}, t)}
\end{aligned}
$$

which can be written as $\langle f \mid \eta\rangle$ for brevity. For turbulent reacting flows, a density-weighted FDF and conditional filtered mean are considered, which are defined as:

$$
\tilde{p}(\eta)=\frac{\langle\rho \mid \eta\rangle}{\bar{\rho}} p(\eta), \quad \widetilde{\langle f \mid \eta\rangle}=\frac{\langle\rho f \mid \eta\rangle}{\langle\rho \mid \eta\rangle}
$$


For the purpose of a priori DNS assessment of LES models, the DNS data has been explicitly LES filtered using a Gaussian filter kernel,

$$
G(\mathbf{x})=\left(\frac{6}{\pi \Delta^{2}}\right)^{3 / 2} \exp \left(-\frac{6 \mathbf{x} \cdot \mathbf{x}}{\Delta^{2}}\right)
$$

so that the LES filtered values of a variable $f$ can be given as $\bar{f}(\mathbf{x})=\int_{V} f\left(\mathbf{x}^{\prime}\right) G\left(\mathbf{x}-\mathbf{x}^{\prime}\right) \mathrm{d} V^{\prime}$ (Pope 2000). In order to evaluate the conditionally filtered values according to the definition given in Eq. 2, it is necessary to numerically approximate the $\delta$-function as $\delta(\xi-\eta) \approx(n / \sqrt{2 \pi}) \exp \left[-0.5(\xi-\eta)^{2} n^{2}\right]$ (Pope 2000) where the parameter $n$ determines the sharpness of this numerical approximation of the $\delta$-function. It has been found that the results become independent to the choice of $n$ for $n>80$ and, accordingly, $n=80$ was chosen for the current analysis.

\subsection{SDR Modelling}

In LES, the filtered SDR is commonly modelled as the sum of a resolved and a sub-grid scale (SGS) part:

$$
\widetilde{N}_{\xi}=\tilde{D} \nabla \tilde{\xi} \cdot \nabla \tilde{\xi}+\widetilde{N}_{\xi, \mathrm{sgs}}
$$

where the latter requires modelling. A model for the SGS scalar dissipation rate typically used for gaseous diffusion flames is (Branley and Jones 2001):

$$
\widetilde{N}_{\xi, \mathrm{sgs}}=\frac{1}{2} C_{N} \frac{v_{\mathrm{sgs}}}{\Delta^{2}} \widetilde{\xi^{\prime \prime 2}}
$$

where $v_{\text {sgs }}$ is the SGS kinematic viscosity and $C_{N}$ is a model constant to be determined. This model is based on a linear relaxation assumption. A wide range of values has been used so far for the model constant $C_{N}$, as used in Eq. 6 or equivalent formulations, stretching from 4 (Ihme and See 2010; Chen et al. 2017) to 20 (Labahn and Devaud 2016) and to 42 (Garmory and Mastorakos 2011; Zhang et al. 2015; Giusti and Mastorakos 2017; Sitte and Mastorakos 2019). The work by Garmory and Mastorakos (2011) recommended the value 42 as the result of an ad-hoc calibration against Sandia Flame F data where $C_{N}$ was adjusted to improve the agreement with the measurements on the mean conditional SDR. It should be noted that this flame is very sensitive to boundary conditions. The present study provides further assessment of this parameter.

The model in Eq. 6 is often used in combination (Zhang et al. 2015) with an algebraic model for the SGS mixture fraction variance based on the equilibrium assumption, i.e. production equals dissipation of scalar variance, which relates the production term of the variance with the scalar dissipation rate (Pierce and Moin 1998; Branley and Jones 2001):

$$
\widetilde{\xi^{\prime \prime 2}}=C_{v} \Delta^{2} \nabla \tilde{\xi} \cdot \nabla \tilde{\xi}
$$

where $C_{v}$ is a model constant typically equal to 0.09 (Branley and Jones 2001). By combining Eqs. 6 and 7, the sub-grid model constants can be related as:

$$
C_{N}=\frac{2}{C_{v} \mathrm{Sc}_{t}}
$$


Similar arguments have been made by Navarro-Martinez et al. (2005) and Pitsch (2006). Equation 8 can be used to determine the theoretical value of a constant when all the other parameters are fixed, under the equilibrium and linear relaxation assumptions.

\subsection{Conditionally-Filtered SDR Modelling}

The conditional filtered SDR requires further modelling. One modelling possibility lies in the numerical inversion of the integral, looking for a best-fit solution (Bushe and Steiner 1999),

$$
\tilde{N}_{\xi}=\int_{0}^{1} \widetilde{\left\langle N_{\xi} \mid \eta\right\rangle} \tilde{p}(\eta) \mathrm{d} \eta
$$

which is, however, computationally expensive and induces smoothing.

In most practical LES-CMC applications other models are used. In CMC, it is usually assumed that the conditional moments vary more smoothly over larger distance then conventionally filtered quantities and a significantly wider filter is used for conditional quantities than the LES filter size, $\Delta_{\mathrm{cmc}} \gg \Delta$. Using the notation from Triantafyllidis and Mastorakos (2010), hereafter variables filtered at the CMC resolution are indicated by a star to distinguish them from variables filtered at the LES resolution. For instance, $\left.\widetilde{\left\langle N_{\xi} \mid \eta\right\rangle}\right\rangle^{*}$ represents the conditional SDR at the CMC resolution and $G^{*}\left(\mathbf{x}-\mathbf{x}^{\prime}\right)$ is the filter corresponding to the filter width $\Delta_{\text {cmc }}$. Mapping of a conditional variable, such as the SDR, from the LES resolution to the CMC resolution is evaluated as follows (Triantafyllidis and Mastorakos 2010):

$$
{\widetilde{\left\langle N_{\xi} \mid \eta\right\rangle}}^{*}=\frac{\int_{V} \bar{\rho} \widetilde{\left\langle N_{\xi} \mid \eta\right\rangle} \tilde{p}(\eta) G^{*}\left(\mathbf{x}-\mathbf{x}^{\prime}\right) \mathrm{d} V^{\prime}}{\int_{V} \bar{\rho} \widetilde{p}(\eta) G^{*}\left(\mathbf{x}-\mathbf{x}^{\prime}\right) \mathrm{d} V^{\prime}}
$$

Navarro-Martinez et al. (2005) proposed a simpler approach than the numerical inversion of the integral for the modelling of the conditional SDR, assuming a low sub-grid variance:

$$
{\widetilde{\left\langle N_{\xi} \mid \eta\right\rangle}}^{*}=\left\langle\tilde{N}_{\xi} \mid \eta\right\rangle^{*}
$$

This approach can be interpreted as neglecting variations of mixture fraction and $N_{\xi}$ in the LES sub-grid scale. The conditional SDR at the level of the CMC resolution, $\left\langle N_{\xi} \mid \eta\right\rangle^{*}$, is obtained by volume averaging conditional on the LES filtered mixture fraction (Eq. 10).

Alternatively, Triantafyllidis et al. (2009) and Kim and Pitsch (2005) used a bell shape for the conditional SDR, which is typically done in RANS. The bell shape for the conditional SDR is obtained from the amplitude mapping closure (AMC) model (O'Brien and Jiang 1991):

$$
\begin{aligned}
\left\langle\widetilde{N_{\xi} \mid \eta}\right\rangle_{\mathrm{amc}} & =N_{0} F_{N}(\eta), \\
F_{N}(\eta) & =\exp \left(-2\left[\operatorname{erf}^{-1}(2 \eta-1)\right]^{2}\right), \\
N_{0} & =\frac{\widetilde{N}_{\xi}}{\int_{0}^{1} F_{N}(\eta) \tilde{p}(\eta) \mathrm{d} \eta}
\end{aligned}
$$

Triantafyllidis and Mastorakos (2010) suggested to presume the bell shape for the conditional SDR in combination with a $\beta$-FDF at the LES resolution, while a Favre-FDF weighted volume average is used to obtain the corresponding values at the $\mathrm{CMC}$ resolution 
(Eq. 10). Different options of these models are evaluated in this work to allow a thorough assessment.

Model 1 assumes that the FDF is a $\delta$-function and uses $\widetilde{N}_{\xi}$ from DNS:

$$
{\widetilde{\left\langle N_{\xi} \mid \eta\right\rangle}}^{*}=\frac{\int_{V} \bar{\rho} \tilde{N}_{\xi} \delta(\eta-\tilde{\xi}) G^{*}\left(\mathbf{x}-\mathbf{x}^{\prime}\right) \mathrm{d} V^{\prime}}{\int_{V} \bar{\rho} \delta(\eta-\tilde{\xi}) G^{*}\left(\mathbf{x}-\mathbf{x}^{\prime}\right) \mathrm{d} V^{\prime}}
$$

Model 2, uses a $\beta$-FDF, $\tilde{p}(\eta)=p_{\beta}\left(\eta ; \tilde{\xi}, \widetilde{\xi^{\prime \prime 2}}\right)$ and $\tilde{N}_{\xi}$ from DNS:

$$
{\widetilde{\left\langle N_{\xi} \mid \eta\right\rangle}}^{*}=\frac{\int_{V} \bar{\rho} \widetilde{N}_{\xi} p_{\beta}(\eta) G^{*}\left(\mathbf{x}-\mathbf{x}^{\prime}\right) \mathrm{d} V^{\prime}}{\int_{V} \bar{\rho} p_{\beta}(\eta) G^{*}\left(\mathbf{x}-\mathbf{x}^{\prime}\right) \mathrm{d} V^{\prime}}
$$

Model 3 uses a $\beta$-FDF, $\widetilde{p}(\eta)=p_{\beta}\left(\eta ; \tilde{\xi}, \widetilde{\xi^{\prime \prime 2}}\right)$, and AMC model with $\widetilde{N}_{\xi}$ computed from DNS:

$$
{\widetilde{\left\langle N_{\xi} \mid \eta\right\rangle}}^{*}=\frac{\int_{V} \bar{\rho}\left\langle\widetilde{N_{\xi} \mid \eta}\right\rangle_{\mathrm{amc}} p_{\beta}(\eta) G^{*}\left(\mathbf{x}-\mathbf{x}^{\prime}\right) \mathrm{d} V^{\prime}}{\int_{V} \bar{\rho} p_{\beta}(\eta) G^{*}\left(\mathbf{x}-\mathbf{x}^{\prime}\right) \mathrm{d} V^{\prime}}
$$

Models 1,2 and 3 use $\widetilde{\xi^{\prime \prime 2}}$ and $\widetilde{N}_{\xi}$ from DNS, hence, assuming a perfect model for these LES-filtered quantities. Consequently, comparing the results allows us to assess the effect of the presumed shapes of the FDF and the conditional SDR.

Model $3 a$ and $3 b$ are also computed from Eq. 15 but using a $\beta$-FDF and AMC model evaluated with SGS mixture fraction variance from Eq. 7 and filtered SDR from Eqs. 5 and 6. Model $3 a$ uses $C_{N}=2 /\left(C_{v} \mathrm{Sc}_{t}\right) \approx 55.56$ whereas Model $3 b$ uses the best-fit value.

\section{Results and Discussion}

\subsection{LES-Filtered SDR}

First, the modelling of the filtered SDR is investigated, following the common approach of splitting it in a resolved and a sub-grid scale part (Eq. 5). Since the model constant of the SGS scalar dissipation, $C_{N}$, can be related to other sub-grid model constants through Eq. 8 ,

Fig. 2 Comparison of the SGS mixture fraction variance $\xi^{\prime \prime 2}$ from DNS and the model with $C_{v}=0.09$. Symbols mark different time instances time instances $t^{+} \approx 10(+), 20(\mathrm{x})$ and 30 (o). $\Delta / \delta_{\text {th }} \approx 2.5$

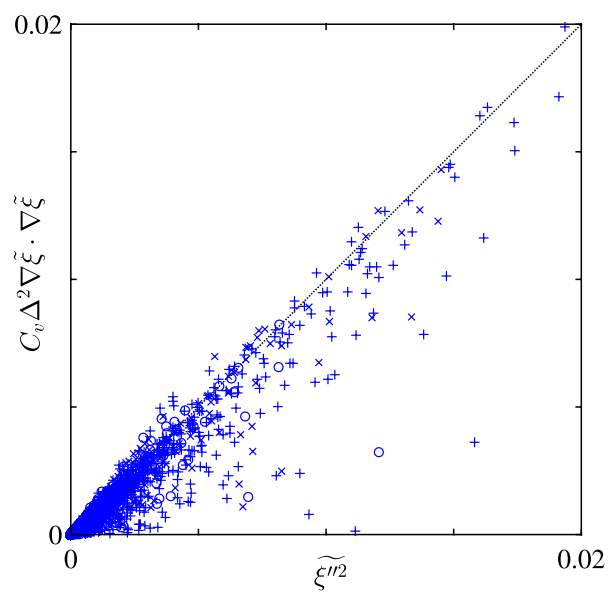


it is instructive to test the accuracy of the sub-grid scalar variance model (Eq. 7). Figure 2 compares the SGS mixture fraction variance from DNS, which is evaluated as $\xi^{\prime \prime 2}=\xi^{2}-\tilde{\xi}^{2}$ (Jiménez et al. 2001), and the prediction from the model with the fixed parameter $C_{v}=0.09$ (Branley and Jones 2001). Good agreement is found independent of location and time with relatively small scatter (correlation coefficient $\approx 0.96$ ). Equally good agreement and approximately the same correlation coefficient was found for LES-filter with the double width, $\Delta / \delta_{\text {th }} \approx 5$. In general, this assessment shows that the algebraic model for the sub-grid variance yields reasonable results and supports the choice of $C_{v}=0.09$.

Furthermore, assuming that the turbulent Schmidt number is between 0.4 and 0.7, Eq. 8 suggests a value for the SDR model parameter $C_{N}$ in the range from $\approx 32$ to 56 . Figure 3 compares the SGS scalar dissipation from DNS with the model predictions (Eq. 6). The value of such constant typically presents a relatively high scatter, that is not dependent on the formulation and persists with dynamic formulations (not shown here). This is further illustrated by Fig. 4 showing optimal value for $C_{N}$, computed after re-arranging the model, given that $\widetilde{N}_{\xi}$ and $v_{\text {sgs }}$ are known: $C_{N}$ covers a wide range of values while, typically, lower values seems to occur in the hot regions engulfed by the flame. In Fig. 3, least-square regression lines are included on top of the scatter to better judge the quality of overall agreement. The DNS results were obtained subtracting the resolved part from the total filtered value. For the model, the sub-grid turbulent viscosity was obtained from the constant Smagorinsky model,

$$
v_{\mathrm{sgs}}=\left(C_{s} \Delta\right)^{2}|\widetilde{\mathbf{S}}|
$$

with the theoretical value $C_{s}=0.17$ (Lilly 1967). It is important to underline that Figs. 2 and 3 report the results for the entire reacting region at different time steps and is, therefore, representative of the jet during the entire ignition process.

Using the model parameter $C_{N} \approx 55.56$ from Eq. 8 with $C_{v}=0.09$ and $\mathrm{Sc}_{t}=0.4$, leads to an over-prediction of the sub-grid SDR. On the other hand, $C_{N}=4$, which is equal to the linear-relaxation model constant commonly used in RANS and also employed in several LES simulations, leads to a significant under-prediction of $\widetilde{N}_{\xi \text {,ggs }}$. The optimal value for the model parameter found in the present study was $C_{N} \approx 18.76$, which allowed for the best fit of the DNS in a least-square sense. Other values have been tested as well; for a lower $C_{s}$ a higher value of $C_{N}$ was required to fit the data (for $C_{s}=0.1$ a best-fit value $C_{N}=54.22$

Fig. 3 Comparison of the sub-grid SDR $\widetilde{N}_{\xi, \text { sgs }}^{+}$from DNS and the model; for $C_{N}=2 /\left(C_{v} \mathrm{Sc}_{t}\right) \approx 55.56$ (blue) at the time instances $t^{+} \approx 10(+)$, 20 (x) and 30 (o) with leastsquare regression lines for each time step (..., -.-, - -) and overall least-square fit (-). For $C_{N}=4$ (red) and the best-fit choice $C_{N}=18.76$ (black) data from all time instances is plotted as small dots. $\Delta / \delta_{\text {th }} \approx 2.5$

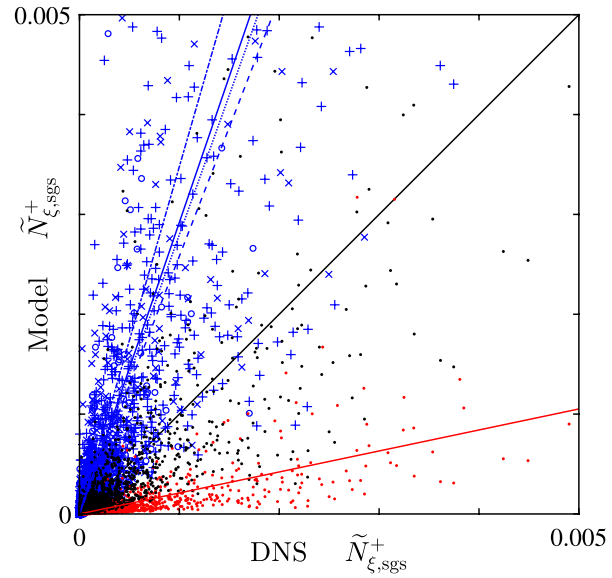


Fig. $4 C_{N}$ computed from DNS as $C_{N}=2 \Delta^{2} N_{\xi, \text { sgs }} / v_{\text {sgs }}$. The region shown is located downstream of the spark location at $x^{+}=15$, corresponding to the instantaneous location of the flame at $t^{+}=10$ (compare with Fig. 1). The value range for $C_{N}$ was clipped at 100. Black lines mark the $T^{+}=0.5$ iso-contour, indicating the position of the flame

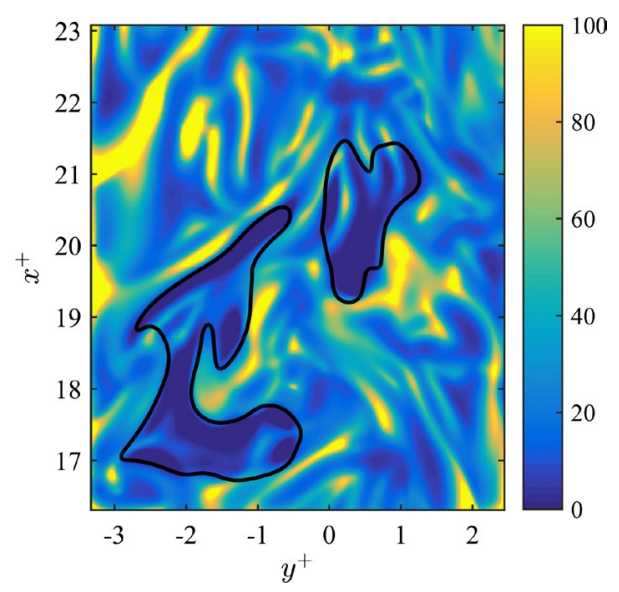

was obtained). Notably, the overall quality of the model prediction does not change significantly in time, even in the ignition process considered. Figure 5 shows results for a larger LES-filter with the double width. The quality of the results is unchanged with correlation coefficient of $\approx 0.78$ and 0.77 for $\Delta / \delta_{\text {th }} \approx 2.5$ and 5 , respectively. These filter widths represent two representative LES filter widths where the flame is fully unresolved which is the case for practical LES simulations. Two different choices of $\Delta / \delta_{\text {th }}>1$ enable us to assess if the model performance is sensitive to the choice of the LES filter width. The current findings suggest that the model performance does not change significantly with LES filter width.

In the next step, the dynamic computations of the Smagorinsky constant $C_{S}$ (Piomelli and Liu 1995) and the model constant for the SGS diffusivity (Pierce and Moin 1998) were performed. Averaging the dynamically computed model constants suggested that $C_{s} \approx 0.110$ and $\mathrm{Sc}_{t} \approx 0.542$ may be more suitable in the present case than the previously used theoretical value. Then a theoretical value of $C_{N} \approx 41.01$ is expected according to Eq. 8. Figure 6 compared the modelled sub-grid SDR, computed with these new model constants to the DNS data. The model performs better with the dynamically computed

Fig. 5 Comparison of the sub-grid SDR $\widetilde{N}_{\xi \text {,sgs }}^{+}$from DNS and the model; for $C_{N}=2 /\left(C_{v} \mathrm{Sc}_{t}\right) \approx 55.56$ (blue), $C_{N}=4$ (red) and $C_{N}=18.76$ (black) at time instant $t^{+} \approx 10$. $\Delta / \delta_{\text {th }} \approx 5$

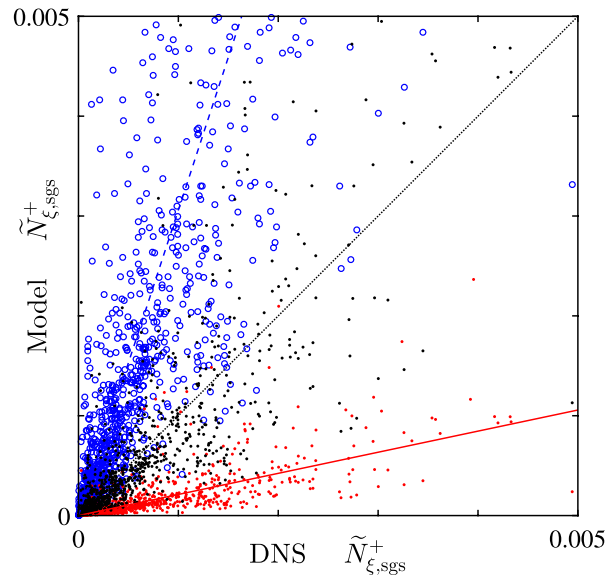


Fig. 6 Comparison of the sub-grid SDR $\widetilde{N}_{\xi \text {,sgs }}^{+}$from DNS and the model using $C_{s} \approx 0.11, \mathrm{Sc}_{t} \approx 0.542$ and $C_{N}=2 /\left(C_{v} \mathrm{Sc}_{t}\right) \approx 41.01$. The dashed blue line marks the least square regression line. $\Delta / \delta_{\text {th }} \approx 2.5$

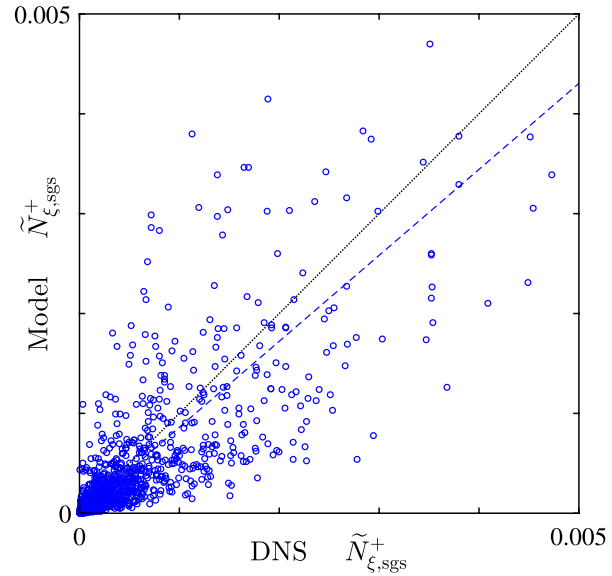

model constants and the theoretical value for $C_{N}$, as far as the scaling of the SDR is concerned in a least-square sense.

Finally, the result of the modelling on the filtered scalar dissipation rate, including the resolved and the sub-grid contribution, is investigated. In Fig. 7, the closure of the total SDR investigated above (Eqs. 5, 6, denoted as linear relaxation model) is compared to the closure (Pierce and Moin 1998; Girimaji and Zhou 1996),

$$
\tilde{N}_{\xi}=\left(\tilde{D}+D_{\text {sgs }}\right) \nabla \tilde{\xi} \cdot \nabla \tilde{\xi}
$$

which is often used when the variance equation is not solved (hereafter denoted as equilibrium models). The comparison was made using the dynamically computed model parameters, $C_{s}=0.11, \mathrm{Sc}_{t}=0.542$ for both models and $C_{N}=41.01$. The sub-grid scale diffusivity was computed as $D_{\text {sgs }}=v_{\text {sgs }} / \mathrm{Sc}_{t}$. The overall performance of both models is similar. For a small number of points the equilibrium model under-predicts the SDR. Note that, both models include the same term, $\tilde{D} \nabla \tilde{\xi} \cdot \nabla \tilde{\xi}$. If the sub-grid variance in the relaxation model was closed with Eq. 7, both models would yield the same result. Notably, the good

Fig. 7 Comparison of the total SDR computed from the linear relaxation model, Eqs. 5 and 6 (o and ...), and the equilibrium model, Eq. 17 (+ and - -); also shown are least-square fit lines, dotted and dashed, respectively. $\Delta / \delta_{\text {th }} \approx 2.5$

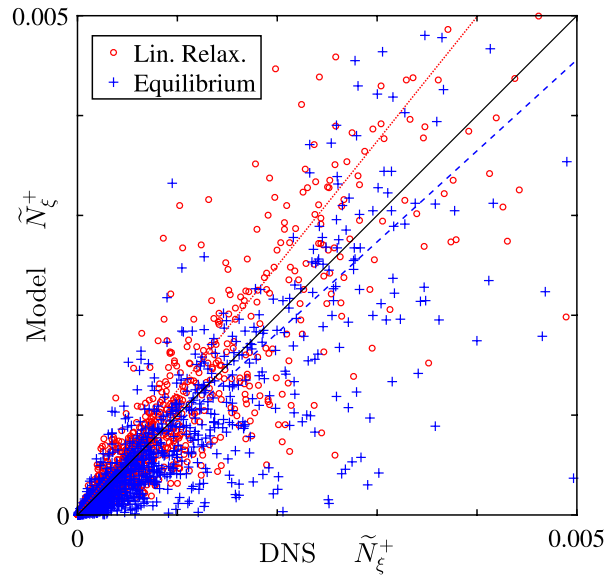


performance of the equilibrium model implies that the modelled production and dissipation terms of the SGS mixture fraction variance equation are of similar size. This is not obvious in the present case, but it has been confirmed that the exact LES-filtered production term, $-2\left[\bar{\rho}\left(\widetilde{u_{i} \xi}-\tilde{u}_{i} \tilde{\xi}\right)\right] \partial \tilde{\xi} / \partial x_{i}$, and the dissipation term, $-2 \overline{\rho D\left(\partial \xi / \partial x_{i}\right)^{2}}+2 \bar{\rho} \tilde{D}\left(\partial \tilde{\xi} / \partial x_{i}\right)^{2}$, directly evaluated from DNS were of the same order of magnitude (not shown), while being the leading order terms in the SGS mixture fraction variance equation.

\subsection{Conditionally Filtered SDR}

Figure 8 compares the conditionally filtered SDR at one location in the middle plane, selected to show the typical behaviour of the models. The DNS result was directly evaluated from DNS data according to Eq. 2. Here, perfect modelling of $\widetilde{\xi}^{\prime \prime 2}$ and $\widetilde{N}_{\xi}$ is assumed, evaluating these variables from DNS, to compare Models 1, 2 and 3. This permits us to assess the presumed shapes of the FDF and the conditional SDR. Models 1 ( $\delta$-PDF and local conditional independence of $N_{\xi}$ ) and 3 ( $\beta$-PDF and presumed bell-curve for $\widehat{\left\langle N_{\xi} \mid \eta\right\rangle}$ locally) predict the conditional SDR reasonably well. In contrast, Model 2 ( $\beta$-PDF and local conditional independence of $N_{\xi}$ ) significantly over-predicts the scalar dissipation for low and high $\eta$. In Fig. 8, the flammability limits are indicated. It is interesting to note that Models 1, 2 and 3 behave similarly in the region where the reaction zone of the flame is located.

The modelling approach based on the $\beta$-PDF and the AMC model is further investigated in the case where $\widetilde{N}_{\xi}$ is modelled. Figure 9 compares the conditional SDR, computed according to Model 3, for different values of $C_{N}$. As could already be seen from Fig. 3, using $C_{N}=2 /\left(\mathrm{Sc}_{t} C_{v}\right) \approx 55.56$ significantly over-predicts the conditional SDR. Model 3b shows the best prediction with the present modelling approach, assuming the optimal parameter $C_{N} \approx 18.76$. This shows that the large scatter observed in Fig. 3 does not have a significant negative effect on $\left\langle\widetilde{N_{\xi} \mid \eta}\right\rangle$ at the level of CMC. These results do not change qualitatively at other spatial locations.

In order to assess the overall performance of the models, we define the square-error of a model in $\eta$-space:

Fig. 8 Non-dimensional conditional SDR from DNS and Models 1,2 and 3 at $\left(x^{+}, y^{+}\right)=(20,1)$ and $t^{+}=10$. Vertical lines mark the stoichiometric mixture fraction, lean and rich flammability limits, $\xi_{\mathrm{st}}=0.317, \xi_{\mathrm{l}}=0.19$ and $\xi_{\mathrm{r}}$ $=0.49$

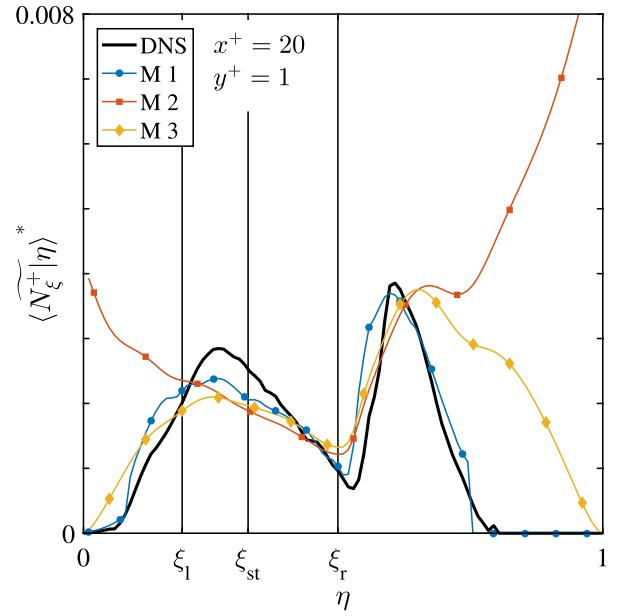


Fig. 9 Sensitivity of Model 3 to the model parameter $C_{N}$. Model $3 \mathrm{a}$ with the theoretical value $C_{N}=55.56$ and Model $3 \mathrm{~b}$ with the best-fit value $C_{N}=18.76$. Location $\left(x^{+}, y^{+}\right)=(20,1)$ and time $t^{+}=10$. Vertical lines mark the lean and the rich flammability limits and the stoichiometric mixture fraction values

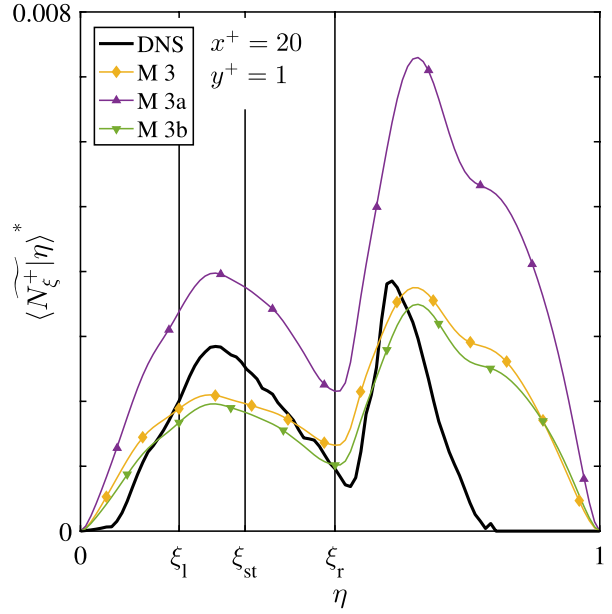

$$
\left({\widetilde{\left\langle N_{\xi} \mid \eta\right\rangle_{\mathrm{M}}}}^{*}-{\widetilde{\left\langle N_{\xi} \mid \eta\right\rangle_{\mathrm{DNS}}}}^{*}\right)^{2}
$$

The overall performance of Models 1,2 and 3 as well as the sensitivity study to $C_{N}$ with Models $3 \mathrm{a}$ and $3 \mathrm{~b}$ is visualised together in Fig. 10, showing the volume-integrated squareerror of the models in $\eta$-space, which generally confirms the observations that had been made for a single location in Figs. 8 and 9.

In the next step, the local variations of the accuracy of the models are assessed. For this purpose, we define the following measure of a local modelling error, which consists of PDF-integrated square error, appropriately normalised:

Fig. 10 Volume-averaged squared error of the modelled $\left\langle N_{\xi}^{+} \mid \eta\right\rangle$ relative to the effective conditional mean from DNS. Vertical lines mark the lean and the rich flammability limit

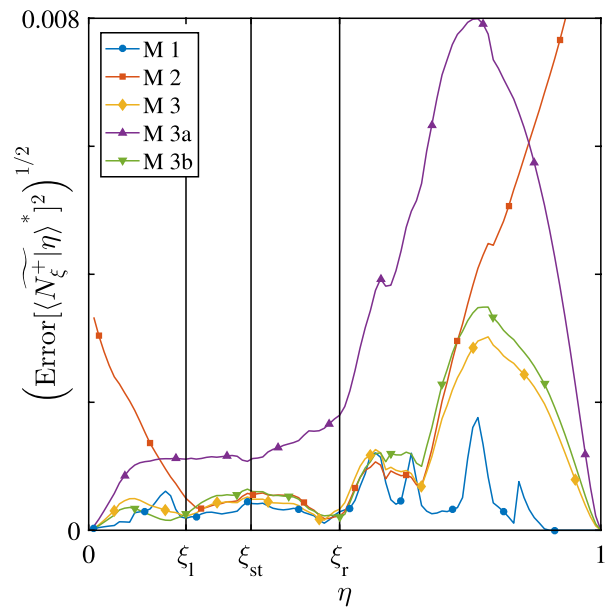



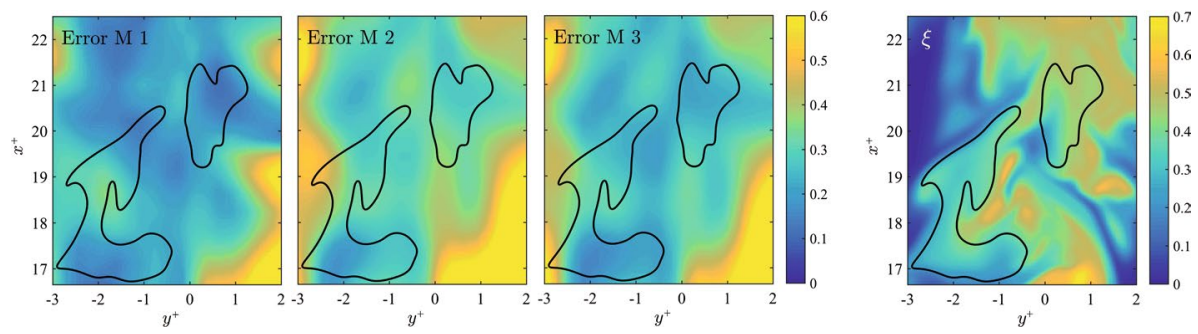

Fig. 11 Local modelling error (Eq. 19) for Models 1, 2 and 3, and the field of the mixture fraction. The region corresponds to the location of the flame at $t^{+}=10$ (compare with Fig. 1. Black lines mark the $T^{+}=0.5$ iso-contour indicating the position of the flame

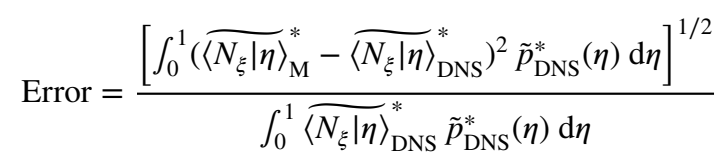

This error metric is shown in Fig. 11 for Models 1, 2 and 3, and the field of the mixture fraction is shown for better interpretation. For Model 1 the errors are generally lowest, with similar performance for Model 3 in the vicinity of the flame; for Model 2 the local error is larger than for the other models in the entire domain. This confirms the observations regarding the model performances for a single point presented in Fig. 8 to the entire domain in Fig. 11. Furthermore, it is apparent from the predictions of Models 2 and 3 that presume $\beta$-PDFs perform worse in the regions outside of the main jet where large portions of unmixed fluid exist.

\section{Conclusions}

Models for the filtered and conditionally-filtered SDR for large-eddy simulations of turbulent non-premixed flames have been assessed for the first time against DNS data for an igniting three-dimensional planar jet flame.

Results demonstrate that the algebraic closure of the sub-grid scale mixture fraction variance based on equilibrium assumption provides a good agreement with the DNS dataset showing the reliability of such model also in an ignition event and independently of the LES filter size. The prediction of the filtered SDR with linear relaxation assumption for the sub-grid scale contribution requires adjustment of the constant that relates the subgrid SDR to the sub-grid mixture fraction variance. The value of the optimal constant of the linear relaxation model for the SGS part of the SDR is above 4 and in agreement with theoretical derivation. A value of such constant one order of magnitude higher than typical values used in Reynolds-averaged approaches is recommended when LES simulations are performed.

The presumed $\beta$-function is generally a good approximation of the sub-grid mixture fraction FDF in the entire domain and throughout the ignition event. Models for conditional SDR based on presumed FDF and AMC model work reasonably well despite the simplicity of the formulation and underlying assumptions. 
The present study focuses on micro-mixing statistics of a passive scalar (i.e. mixture fraction), which is unlikely to be affected by the choice of the chemical mechanism. However, the present findings based on reduced chemical mechanism need to be subsequently validated in the context of 3D DNS with detailed chemistry and transport, which will form the basis of future analysis for a more comprehensive analysis.

In terms of generality, previous analyses for premixed turbulent flames suggested that the model parameters for SDR closures attain asymptotic values for relatively small values of turbulent Reynolds number and that SDR modelling was not sensitive to the geometric configuration as long as the mean curvature remained small. Nevertheless, the present modelling methodologies need to be assessed for higher values of Reynolds number and in configurations with a small mean radius for further validations.

Acknowledgements We are grateful to Rocket HPC of Newcastle and Cirrus Tier-2 system for access to computational resources.

Funding This research received funding from EPSRC and UKCTRF (EP/R029369/1).

\section{Compliance with Ethical Standards}

Conflict of interest The authors declare that they have no conflict of interest.

Open Access This article is licensed under a Creative Commons Attribution 4.0 International License, which permits use, sharing, adaptation, distribution and reproduction in any medium or format, as long as you give appropriate credit to the original author(s) and the source, provide a link to the Creative Commons licence, and indicate if changes were made. The images or other third party material in this article are included in the article's Creative Commons licence, unless indicated otherwise in a credit line to the material. If material is not included in the article's Creative Commons licence and your intended use is not permitted by statutory regulation or exceeds the permitted use, you will need to obtain permission directly from the copyright holder. To view a copy of this licence, visit http://creativecommons.org/licenses/by/4.0/.

\section{References}

Ahmed, S.F., Mastorakos, E.: Spark ignition of lifted turbulent jet flames. Combust. Flame 146(1), $215-231$ (2006). https://doi.org/10.1016/j.combustflame.2006.03.007

Akselvoll, K., Moin, P.: Large-eddy simulation of turbulent confined coannular jets. J. Fluid Mech. 315, 387-411 (1996). https://doi.org/10.1017/S0022112096002479

Bibrzycki, J., Poinsot, T .:Reduced chemical kinetic mechanisms for methane combustion in O2/N2 and O2/ CO2 atmosphere. Work note ECCOMET WN/CFD/10/17, CERFACS (2010)

Bilger, R.W.: Some aspects of scalar dissipation. Flow Turbul. Combust. 72(2), 93-114 (2004). https://doi. org/10.1023/B:APPL.0000044404.24369.f1

Branley, N., Jones, W.P.: Large eddy simulation of a turbulent non-premixed flame. Combust. Flame 127(1), 1914-1934 (2001). https://doi.org/10.1016/S0010-2180(01)00298-X

Bushe, W.K., Steiner, H.: Conditional moment closure for large eddy simulation of nonpremixed turbulent reacting flows. Phys. Fluids 11(7), 1896-1906 (1999). https://doi.org/10.1063/1.870052

Chakraborty, N., Mastorakos, E.: Numerical investigation of edge flame propagation characteristics in turbulent mixing layers. Phys. Fluids 18(10), 105103 (2006). https://doi.org/10.1063/1.2357972

Chakraborty, N., Swaminathan, N.: Reynolds number effects on scalar dissipation rate transport and its modeling in turbulent premixed combustion. Combust. Sci. Technol. 185(4), 676-709 (2013). https:// doi.org/10.1080/00102202.2012.741635

Chakraborty, N., Hesse, H., Mastorakos, E.: Effects of fuel lewis number on localised forced ignition of turbulent mixing layers. Flow Turbul. Combust. 84(1), 125 (2009). https://doi.org/10.1007/s1049 4-009-9231-1 
Chen, Z., Ruan, S., Swaminathan, N.: Large eddy simulation of flame edge evolution in a spark-ignited methane-air jet. Proc. Combust. Inst. 36(2), 1645-1652 (2017). https://doi.org/10.1016/j.proci .2016.06.023

Devaud, C.B., Bilger, R.W., Liu, T.: A new method of modeling the conditional scalar dissipation rate. Phys. Fluids 16(6), 2004-2011 (2004). https://doi.org/10.1063/1.1699108

Gao, Y., Chakraborty, N.: Modeling of Lewis number dependence of scalar dissipation rate transport for large eddy simulations of turbulent premixed combustion. Numer. Heat Transf. Part A Appl. 69(11), 1201-1222 (2016). https://doi.org/10.1080/10407782.2015.1125732

Garmory, A., Mastorakos, E.: Capturing localised extinction in Sandia Flame F with LES-CMC. Proc. Combust. Inst. 33(1), 1673-1680 (2011). https://doi.org/10.1016/j.proci.2010.06.065

Girimaji, S.S.: On the modeling of scalar diffusion in isotropic turbulence. Phys. Fluids 4(11), 2529-2537 (1992). https://doi.org/10.1063/1.858440

Girimaji, S.S., Zhou, Y.: Analysis and modeling of subgrid scalar mixing using numerical data. Phys. Fluids 8(5), 1224-1236 (1996). https://doi.org/10.1063/1.868894

Giusti, A., Mastorakos, E.: Detailed chemistry LES/CMC simulation of a swirling ethanol spray flame approaching blow-off. Proc. Combust. Inst. 36(2), 2625-2632 (2017). https://doi.org/10.1016/j.proci .2016 .06 .035

Hesse, H., Chakraborty, N., Mastorakos, E.: The effects of the lewis number of the fuel on the displacement speed of edge flames in igniting turbulent mixing layers. Proc. Combust. Inst. 32(1), 1399-1407 (2009). https://doi.org/10.1016/j.proci.2008.06.065

Ihme, M., See, Y.C.: Prediction of autoignition in a lifted methane/air flame using an unsteady flamelet/ progress variable model. Combust. Flame 157(10), 1850-1862 (2010). https://doi.org/10.1016/j. combustflame.2010.07.015

Jenkins, K.W., Cant, R.S.: Direct numerical simulation of turbulent flame kernels. In: Knight, D., Sakell, L. (eds.) Recent Advances in DNS and LES, pp. 191-202. Springer, Netherlands, Dordrecht (1999)

Jiménez, C., Ducros, F., Cuenot, B., Bédat, B.: Subgrid scale variance and dissipation of a scalar field in large eddy simulations. Phys. Fluids 13(6), 1748-1754 (2001). https://doi.org/10.1063/1.1366668

Kaul, C.M., Raman, V.: A posteriori analysis of numerical errors in subfilter scalar variance modeling for large eddy simulation. Phys. Fluids 23(3), 035102 (2011). https://doi.org/10.1063/1.3556097

Kaul, C.M., Raman, V., Balarac, G., Pitsch, H.: Numerical errors in the computation of subfilter scalar variance in large eddy simulations. Phys. Fluids 21(5), 055102 (2009). https://doi. org/10.1063/1.3123531

Kaul, C.M., Raman, V., Knudsen, E., Richardson, E.S., Chen, J.H.: Large eddy simulation of a lifted ethylene flame using a dynamic nonequilibrium model for subfilter scalar variance and dissipation rate. Proc. Combust. Inst. 34(1), 1289-1297 (2013). https://doi.org/10.1016/j.proci.2012.06.079

Kim, S.H., Pitsch, H.: Conditional filtering method for large-eddy simulation of turbulent nonpremixed combustion. Phys. Fluids 17(10), 105103 (2005). https://doi.org/10.1063/1.2084229

Klimenko, A.Y., Bilger, R.W.: Conditional moment closure for turbulent combustion. Prog. Energy Combust. Sci. 25(6), 595-687 (1999). https://doi.org/10.1016/S0360-1285(99)00006-4

Klimenko, A.Y., Pope, S.B.: The modeling of turbulent reactive flows based on multiple mapping conditioning. Phys. Fluids 15(7), 1907-1925 (2003). https://doi.org/10.1063/1.1575754

Kronenburg, A., Bilger, R.W., Kent, J.H.: Computation of conditional average scalar dissipation in turbulent jet diffusion flames. Flow Turbul. Combust. 64(3), 145-159 (2000). https://doi. org/10.1023/A:1009912404132

Labahn, J.W., Devaud, C.B.: Large eddy simulations (LES) including conditional source-term estimation (CSE) applied to two Delft-Jet-in-hot-coflow (DJHC) flames. Combust. Flame 164, 68-84 (2016). https://doi.org/10.1016/j.combustflame.2015.11.002

Lilly, D.K.: The representation of small-scale turbulence in numerical simulation experiments. In: Proceedings of the IBM Scientific Computing Symposium on Environmental Sciences, Yorktown Heights pp. 195-210 (1967)

Namer, I., Ötügen, M.V.: Velocity measurements in a plane turbulent air jet at moderate reynolds numbers. Exp. Fluids 6(6), 387-399 (1988). https://doi.org/10.1007/BF00196484

Navarro-Martinez, S., Kronenburg, A.: Flame stabilization mechanisms in lifted flames. Flow Turbul. Combust. 87(2), 377-406 (2011). https://doi.org/10.1007/s10494-010-9320-1

Navarro-Martinez, S., Kronenburg, A., Di Mare, F.: Conditional moment closure for large eddy simulations. Flow Turbul. Combust. 75(1-4), 245-274 (2005). https://doi.org/10.1007/s10494-005-8580-7

O'Brien, E.E., Jiang, T.: The conditional dissipation rate of an initially binary scalar in homogeneous turbulence. Phys. Fluids A-Fluid 3(12), 3121-3123 (1991). https://doi.org/10.1063/1.858127

Peters, N.: Laminar diffusion flamelet models in non-premixed turbulent combustion. Prog. Energy Combust. 10(3), 319-339 (1984). https://doi.org/10.1016/0360-1285(84)90114-X 
Peters, N., Williams, F.A.: The asymptotic structure of stoichiometric methane-air flames. Combust. Flame 68(2), 185-207 (1987). https://doi.org/10.1016/0010-2180(87)90057-5

Pierce, C.D., Moin, P.: A dynamic model for subgrid-scale variance and dissipation rate of a conserved scalar. Phys. Fluids 10(12), 3041-3044 (1998). https://doi.org/10.1063/1.869832

Piomelli, U., Liu, J.: Large-eddy simulation of rotating channel flows using a localized dynamic model. Phys. Fluids 7(4), 839-848 (1995). https://doi.org/10.1063/1.868607

Pitsch, H.: Large-eddy simulation of turbulent combustion. Annu. Rev. Fluid Mech. 38(1), 453-482 (2006). https://doi.org/10.1146/annurev.fluid.38.050304.092133

Pope, S.B.: PDF methods for turbulent reactive flows. Prog. Energy Combust. Sci. 11(2), 119-192 (1985). https://doi.org/10.1016/0360-1285(85)90002-4

Pope, S.B.: Computations of turbulent combustion: progress and challenges. Symp. (Int) Combust. 23(1), 591-612 (1991). https://doi.org/10.1016/S0082-0784(06)80307-3

Pope, S.B.: Turbulent Flows. Cambridge University Press, Cambridge (2000)

Ren, Z., Pope, S.B.: An investigation of the performance of turbulent mixing models. Combust. Flame 136(1), 208-216 (2004). https://doi.org/10.1016/j.combustflame.2003.09.014

Richardson, E.S., Mastorakos, E.: Numerical investigation of forced ignition in laminar counterflow non-premixed methane-air flames. Combust. Sci. Technol. 179(1-2), 21-37 (2007). https://doi. org/10.1080/00102200600805892

Sitte, M.P., Mastorakos, E.: Large eddy simulation of a spray jet flame using doubly conditional moment closure. Combust. Flame 199, 309-323 (2019). https://doi.org/10.1016/j.combustflame.2018.08.026

Triantafyllidis, A., Mastorakos, E.: Implementation issues of the conditional moment closure model in large eddy simulations. Flow Turbul. Combust. 84(3), 481-512 (2010). https://doi.org/10.1007/s1049 4-009-9226-y

Triantafyllidis, A., Mastorakos, E., Eggels, R.L.G.M.: Large eddy simulations of forced ignition of a nonpremixed bluff-body methane flame with conditional moment closure. Combust. Flame 156(12), 2328-2345 (2009). https://doi.org/10.1016/j.combustflame.2009.05.005

Turquand d'Auzay, C., Chakraborty, N.: The localised forced ignition and early stages of flame development in a turbulent planar jet. Proc Combust Inst, 38 (submitted) (2020)

Turquand d'Auzay, C., Papapostolou, V., Ahmed, S.F., Chakraborty, N.: Effects of turbulence intensity and biogas composition on the localized forced ignition of turbulent mixing layers. Combust. Sci. Technol. 191(5-6), 868-897 (2019a). https://doi.org/10.1080/00102202.2019.1576651

Turquand-d'Auzay, C., Ahmed, U., Pillai, A., Chakraborty, N., Kurose, R.: Statistics of progress variable and mixture fraction gradients in an open turbulent jet spray flame. Fuel 247, 198-208 (2019b). https:// doi.org/10.1016/j.fuel.2019.02.111

Varna, A., Cleary, M., Hawkes, E.: A multiple mapping conditioning mixing model with a mixture-fraction like reference variable. Part 1: Model derivation and ideal flow test cases. Combust. Flame 181, 342353 (2017). https://doi.org/10.1016/j.combustflame.2017.03.016

Vázquez Espí, C., Liñán, A.: Thermal-diffusive ignition and flame initiation by a local energy source. Combust. Theor. Model 6(2), 297-315 (2002). https://doi.org/10.1088/1364-7830/6/2/309

Wacks, D.H., Chakraborty, N.: Statistical analysis of the reaction progress variable and mixture fraction gradients in flames propagating into droplet mist: A Direct Numerical Simulation analysis. Combust. Sci. Technol. 188, 2149-2177 (2016). https://doi.org/10.1080/00102202.2016.1212605

Wandel, A.P.: Influence of scalar dissipation on flame success in turbulent sprays with spark ignition. Combust. Flame 161(10), 2579-2600 (2014). https://doi.org/10.1016/j.combustflame.2014.04.006

Zhang, H., Mastorakos, E.: Prediction of global extinction conditions and dynamics in swirling non-premixed flames using LES/CMC modelling. Flow Turbul. Combust. 96(4), 863-889 (2016). https://doi. org/10.1007/s10494-015-9689-y

Zhang, H., Garmory, A., Cavaliere, D.E., Mastorakos, E.: Large eddy simulation/conditional moment closure modeling of swirl-stabilized non-premixed flames with local extinction. Proc. Combust. Inst. 35(2), 1167-1174 (2015). https://doi.org/10.1016/j.proci.2014.05.052

Zhang, H., Giusti, A., Mastorakos, E.: LES/CMC modelling of ignition and flame propagation in a nonpremixed methane jet. Proc. Combust. Inst. 37(2), 2125-2132 (2019). https://doi.org/10.1016/j.proci .2018.09.031 